\title{
Topological Measures of Connectomics for Low Grades Glioma
}

\author{
Benjamin Amoah ${ }^{1}$ and Alessandro Crimi*2 \\ ${ }^{1}$ Lancaster University, Lancaster, UK \\ ${ }^{2}$ Istituto Italiano di Tecnologia, Genoa, Italy
}

\begin{abstract}
Recent advancements in neuroimaging have allowed the use of network analysis to study the brain in a system-based approach. In fact, several neurological disorders have been investigated from a network perspective. These include Alzheimer's disease, autism spectrum disorder, stroke, and traumatic brain injury. So far, few studies have been conducted on glioma by using connectome techniques. A connectome-based approach might be useful in quantifying the status of patients, in supporting surgical procedures, and ultimately shedding light on the underlying mechanisms and the recovery process.

In this manuscript, by using graph theoretical methods of segregation and integration, topological structural connectivity is studied comparing patients with low grade glioma to healthy control. These measures suggest that it is possible to quantify the status of patients pre- and post-surgical intervention to evaluate the condition.

Keywords: Autism Spectrum Disorder, Autism Spectrum Disorder, Fractional Anisotropy, Functional Connectivity, Cluster Coefficient
\end{abstract}

\section{Introduction}

Glioma is the most common type of primary brain tumor which arises from glial cells. It is considered responsible for approximately 13000 deaths in the United States and more than 14000 in Europe each year [1]. It is considered one of the most aggressive types of cancer especially in its advanced stage termed glioblastoma multiforme (GBM). Tumor resection is the most effective therapy though generally complemented by chemo and radio therapies [2].

*alecrimi@alice.it; Corresponding author 
Nevertheless, resection of brain tumors involving relevant cortical areas is still a challenging task, as preservation of neuronal functions after surgery remains the goal [2]. Several studies have shown the potentiality of magnetic resonance imaging (MRI) in glioma patients for identifying pre-operatively their relationship with eloquent cortical areas, but individual significant variations in fiber structures and functional MRI (fMRI) activations have been reported $[3,4,2]$. Diffusion-tensor imaging (DTI) is used to track fibers combined with cortical stimulation as intra-operative support to preserve cognitive and motor functions, though this analysis might be subjective [5]. Moreover, analysis of functional activations highlights only the activations related to the task, but not the interaction among areas or the effect among the overall brain.

A Connectome is the complete set of all neural connections of the human brain which can be structural or functional [6]. The human connectome has recently gained attention for its importance and possible implications for neuroscience as well as clinical neurology and psychology. It has already been used for studying stroke, autism spectrum disorders (ASD), Alzheimer's disease, schizophrenia, and other pathologies $[7,8]$. However, connectome analysis has not been extensively used for glioma patients. As for stroke, it is expected that specific cognitive and behavioral functions are not localized to anatomically restricted areas, but widespread across the neural networks of the injured brain, and specific symptoms are not necessarily localized in specific brain regions [9]. Hence, it can be hypothesized that connectomics can help to study cortical reorganization, functional recovery after resection, and help planning surgical interventions. Briganti et al. studied the functional connectivity of glioma affected patients by using a verb-generation task acquisition, and noticed that the patients had a statistically significant reduced degree of functional connectivity in the language related regions compared to healthy control [10]. Similarly, in another study functional connectivity exhibited chaotic changes in glioma patients compared to control correlating with language deficits [11]. It was also noticed that patients with gliomas have altered functional connectivity of the default mode network, and this was related to tumor grade, position and post-surgical status [12]. 
In this manuscript, topological measures are used to quantify the level of segregation and integration comparing low grade glioma patients and healthy control.

\section{Method}

An individual network measure may characterize one or several aspects of global and local brain connectivity. This study starts creating the tractography of the brain from DTI for patients and control, generating the related structural connectome, on which topological measures are computed to compare the two groups: low grade glioma patients and healthy control subjects.

Tractographies for all subjects have been generated processing DTI data with the Python library Dipy [13]. In particular, a deterministic algorithm called Euler Delta Crossings [13] has been used stemming from 2,000,000 seed-points and stopping when the fractional anisotropy (FA) was smaller than $<0.1$. Tracts shorter than $30 \mathrm{~mm}$ or in which a sharp angle occurred have been discarded. Linear registration has been applied between the automated anatomical labeling (AAL) atlas [14] and the first volume of the DTI acquisition by using linear registration with 12 degrees of freedom. Counting the fibers starting and ending in all $r=90$ regions of the AAL atlas, a structural connectivity matrix of $r \times r$ elements is constructed for each subject. Connections with less than 4 fibers are neglected, and the matrix is afterwards binarized.

Once the connectome is constructed, the glioma patients and control subjects were characterized by the most common graph-topological measures. Topological measures are divided into measures of segregation and integration. Segregation measures are representations of densely connected network communities, while integration measures are related to network hubs that are rich in connections between the communities [15]. Simulations on artificial networks demonstrated that as the connectivity gradually changes from an ordered lattice to a pseudo-random network, perturbational integration decreases, and perturbational seg- 
regation increases [15]. However these decreases and increases are not easily quantifiable as different diseases might affect in different ways the brain or relevant nodes of it [16]. For this study, we chose the two most common measures of segregation (the Louvain modularity and clustering coefficient) and integration (characteristic path length and global efficiency) [17] computed for the n nodes of each graph.

The modularity of a network is the degree to which the network may be subdivided into non-overlapping groups. The Louvain algorithm is known for its efficiency in producing partitioned communities, and it is applicable to weighted and unweighted graphs. For weighted graphs, modularity is defined as

$$
Q=\frac{1}{2 m} \sum_{i j}\left[A_{i j}-\frac{k_{i} k_{j}}{2 m}\right] \delta\left(c_{i}, c_{j}\right),
$$

where $t_{i}$ and $k_{i}$ is respectively the number of triangles around a node $\mathrm{i}$, and the degree of the node $i$. It measures how much neighbors of a node are connected to each other. In the results, the mean value across the nodes are reported. The characteristic path length $d_{i j}$ is the average shortest path length in the network between each node $i$ and $j$. The efficiency measure is given by the average inverse shortest path length. It can be computed globally or limited to the neighborhood of a node defining the local efficiency. Global efficiency is defined as

$$
E=\frac{1}{n} \sum_{i \in N} \frac{\sum_{j \in N, j \neq i} d_{i j}^{-1}}{n-1}
$$

Given the measures as features for representing the two populations of glioma patients and control, p-values were generated from a two-sample t-tests performed on each metric with the goal to assess difference between the two groups. 


\section{Data and Experimental Settings}

The neuroimage data for the patients are from The Cancer Imaging Archive (TCIA) (http: //cancerimagingarchive.net), which is a large and growing archive, comprising several hundreds volumes in different modalities. However, diffusion tensor and fMRI data available are very limited or acquired in different protocols. The brain volumes for the low grade glioma patients were acquired with a 1.5 T GE Signa Excite. In particular, the 20 available DTI volumes were acquired with an isotropic voxel-size of $2.6 \mathrm{~mm}$, TR $=17 \mathrm{~s}, \mathrm{TE}=84.6$ ms, and using 26 gradient directions. The mean age of the patients was $45.74 \pm 13.35$ years.

Being no control brain available in TCIA archive, those were matched with the 20 control volumes available from the NKI-Rockland Sample (http://fcon_1000.projects.nitrc. org/indi/pro/nki.html) randomly selected. Those DTI volumes were acquired with a Siemens 1.5 T scanner and isotropic voxel-size of $2 \mathrm{~mm}, \mathrm{TR}=10 \mathrm{~s}$, TE $=91 \mathrm{~ms}$, and using 35 gradient directions. The age of the healthy control was $38 \pm 19.15$ years.

All data had the skull stripped and eddy current correction performed before the tractography construction.

\section{Results}

The results of the network analysis for the two datasets and significance are reported in Table 1 as mean and variance value for the two groups. The last column reports the p-value of the discriminative test between the two groups. An example of resulting connectome for the healthy control subjects is shown in Fig. 1, while Figs. 2 and 3 depict axial slices for two cases subjects. In particular, those are T1 post gadolinium injection, FLAIR, tractography stopping the tracts if the FA was smaller than 0.25, and structural connectome using the tractography constructed stopping the tracts if the FA was smaller than 0.01. 
Table 1. Topological network measures reported as mean and variance. The last column reports the p-value of the discriminative test between the two groups.

\begin{tabular}{|l|l|l|l|}
\hline Features & Low grade glioma patients & Healthy controls & p-value \\
\hline Modularity & $0.491 \pm 0.021$ & $0.438 \pm 0.026$ & $1.15 \times 10^{-7}$ \\
\hline Clustering coefficient & $0.605 \pm 0.012$ & $0.592 \pm 0.017$ & 0.0252 \\
\hline Char. path length & $2.265 \pm 0.058$ & $2.139 \pm 0.054$ & $1.15 \times 10^{-7}$ \\
\hline Global efficiency & $0.512 \pm 0.010$ & $0.540 \pm 0.010$ & $1.13 \times 10^{-8}$ \\
\hline
\end{tabular}

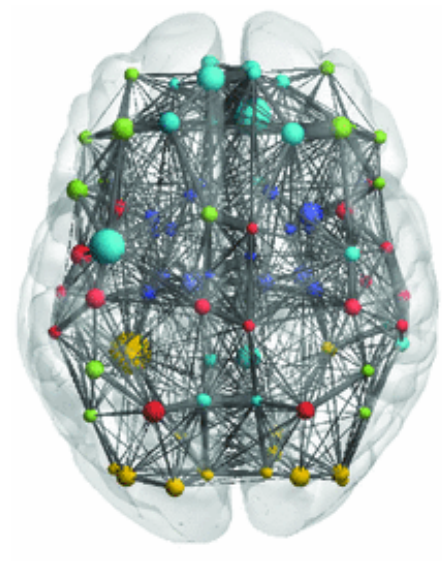

(a)

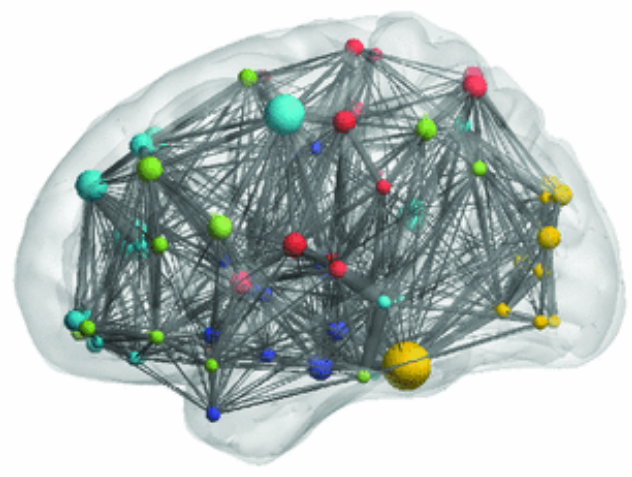

(b)

Figure 1. Axial (a) and sagittal (b) view of a healthy control connectome (no slice). The size of the nodes represents the degree, while the number of tracts connecting the nodes is represented by the stroke of the edges. The tractography for these structural connections is constructed stopping the tracts if the FA was smaller than 0.01. 


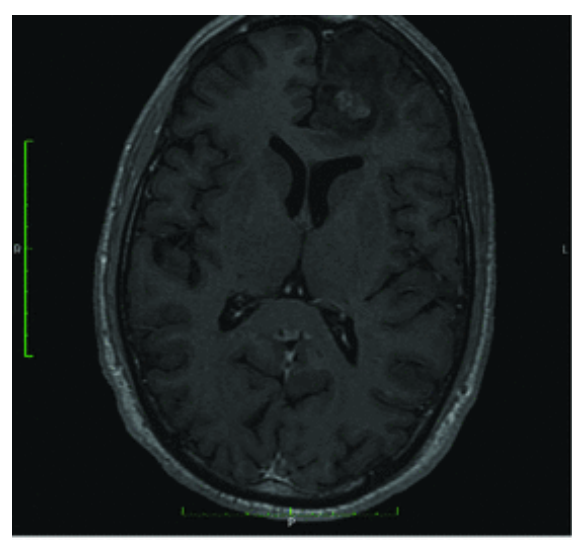

(a)

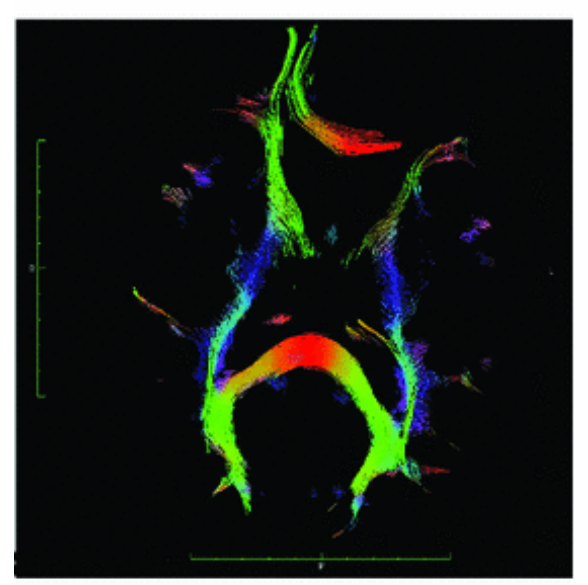

(c)

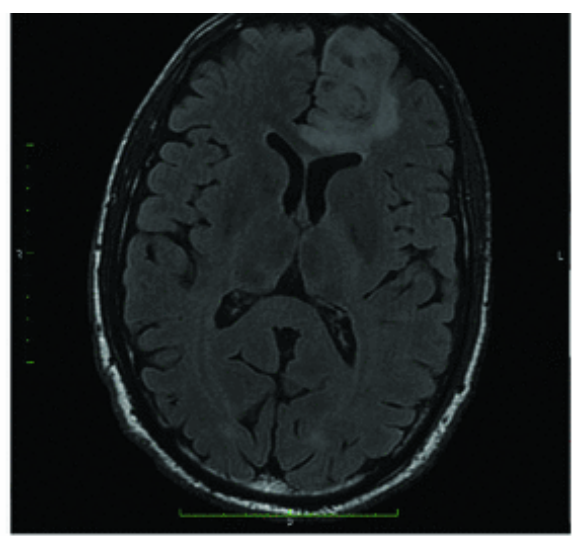

(b)

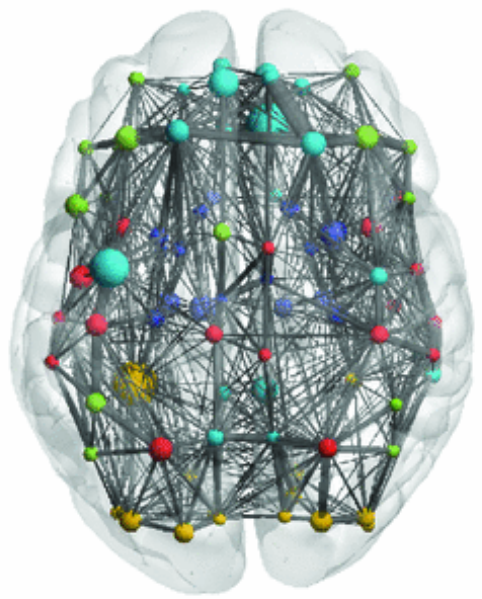

(d)

Figure 2. Presurgical axial slices for one case subjects: (a) T1 post gadolinium injection, (b) FLAIR, (c) tractography stopping the tracts if the FA was smaller than 0.25, and (d) whole structural connectome using the tractography constructed stopping the tracts if the FA was smaller than 0.01 (no slice). 


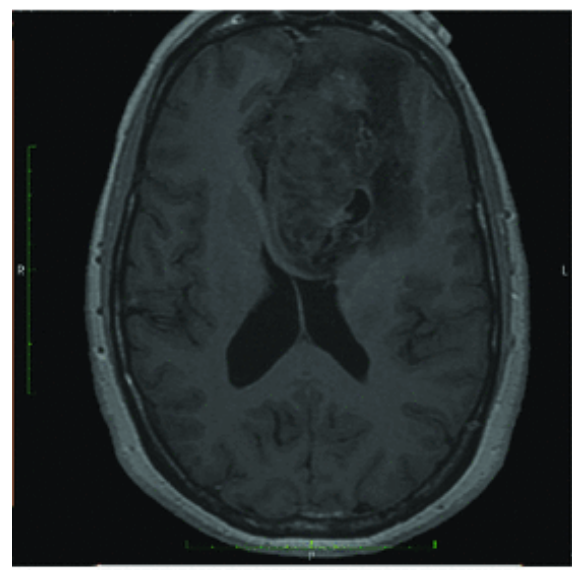

(a)

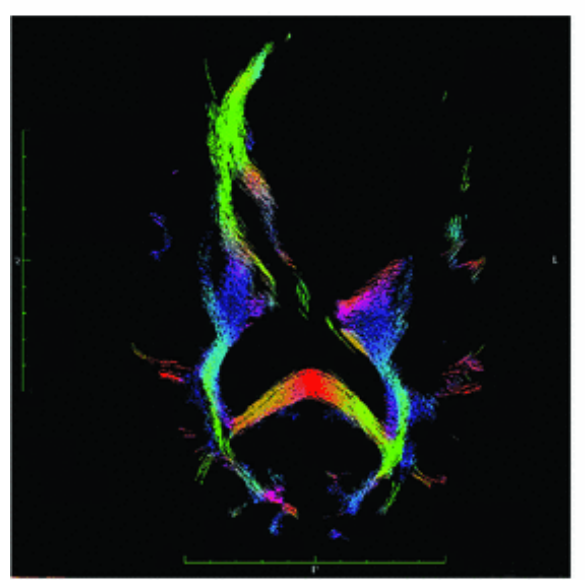

(c)

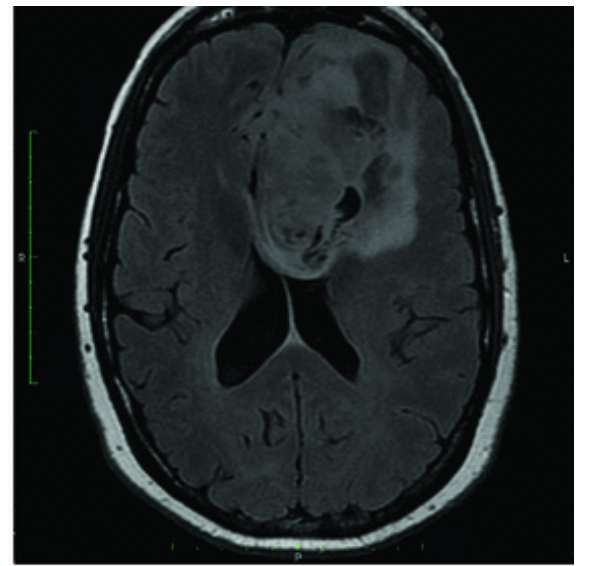

(b)

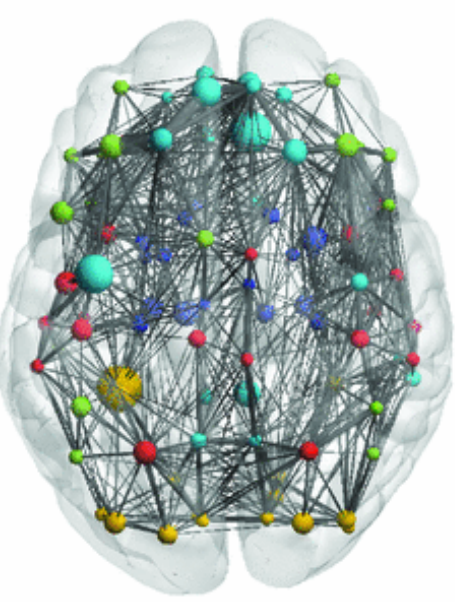

(d)

Figure 3. Presurgical axial slices for one case subjects: (a) T1 post gadolinium injection, (b) FLAIR, (c) tractography stopping the tracts if the FA was smaller than 0.25 , and (d) whole structural connectome using the tractography constructed stopping the tracts if the FA was smaller than 0.01 (no slice) 


\section{Discussions}

The measures of segregation and integration used in this article have been already investigated for other diseases. In a study about ASD, all measures used in functional connectomes showed lower scores in ASD patients compared to healthy control [18]. In a study related to schizophrenia [19], connectomes of patients with psychotic episodes showed larger characteristic path length, but smaller global efficiency and clustering coefficients compared to schizophrenia subject without psychotic episodes. Another topological comparison between connectomes of schizophrenia patients and control subject showed smaller global efficiency and other integration metrics in the patients [20]. Conversely, other studies on schizophrenia showed elevated values of clustering coefficients and small values of characteristic shortest path, suggesting overall more segregated patterns in the network [21]. This lack of agreement can be related to different pre-processing steps, neglected local anatomical differences, or on the selecting criteria of the matching control group [21].

Our results for the case connectomes showed an increased modularity and clustering coefficient, and an increased characteristic path length and reduced related global efficiency compared to the control connectome scores. The increased path length and reduced global efficiency can be hypothetically explained as the destructive effect of low grade glioma being similar to the disconnecting impact of schizophrenia which share the same discrimination between case and control.

No clear difference was noticeable by visual inspection of the connectome, though an atlas with more detailed cortical subdivision might have allowed also this visual difference [22]. However, if the stopping threshold of the FA was moved from 0.01 to 0.25 , the tracts crossing the tumor disappeared. This is a sign of damage in the area. It can hypothesized that due to the low grade of the tumors tracts are still presents and not completely damaged as with glioblastoma which is a brain tumor of higher grade. The main limitation of the study is the limited sample size and also the different type of acquisition for the case and control dataset which might have influenced the detected differences. Therefore, further studies with even 
more consistent datasets are required. Moreover, the measures give an indicative score of the status of the network to potentially correlate with health status, but they are not enough to help during surgical procedures, since population statistics to identify relevant areas or connections has only a relative meaning for pre-surgical planning [23]. However, the study of individual connectomes could be used jointly to existing procedure of cortical activation and stimulation to support surgical decisions.

Despite the registration of the volumes to the atlas was considered properly carried out as noticed by visual inspection, concerns remain about possible influence of the glioma in deforming the brain anatomy. In fact, it can be argued that due to the presence of the tumor some tracts might be pushed ending in a different location than expected by the atlas and therefore corrupting the subsequent analysis. The issue has been argued in tract based spatial statistics studies considering multiple sclerosis lesions, concluding that the misplacement effect is negligible [24]. Conversely, it has been argued that for high grade glioma located near the corticospinal tracts and eloquent areas, it is possible that such an effect has an impact [10]. Being this study mostly focused on the overall topological measures and using only low grade gliomas patients, this issue could be considered negligible, though an analysis with a model which take into account potential displacement is planned as a future work.

\section{Conclusion}

Understanding the brain connectome and dynamic network changes that occur due to tumor can give further information on the status of patients and evaluate rehabilitations. In fact, topological measures appear to differ in terms of segregation and integration in glioma patients compared to healthy control. 


\section{References}

[1] Siegel, R.L., Miller, K.D., Jemal, A.: Cancer statistics, 2016. CA: a cancer journal for clinicians 66(1), 7-30 (2016)

[2] Sanai, N., Berger, M.S.: Glioma extent of resection and its impact on patient outcome. Neurosurgery 62(4), 753-766 (2008)

[3] Fandino, J., Kollias, S.S., Wieser, H.G., Valavanis, A., Yonekawa, Y.: Intraoperative validation of functional magnetic resonance imaging and cortical reorganization patterns in patients with brain tumors involving the primary motor cortex. Journal of neurosurgery 91(2), 238-250 (1999)

[4] Kapsalakis, I.Z., Kapsalaki, E.Z., Gotsis, E.D., Verganelakis, D., Toulas, P., Hadjigeorgiou, G., Chung, I., Fezoulidis, I., Papadimitriou, A., Robinson, J.S., et al.: Preoperative evaluation with fmri of patients with intracranial gliomas. Radiology research and practice 2012 (2012)

[5] Berman, J.I., Berger, M.S., Mukherjee, P., Henry, R.G.: Diffusion-tensor imaging - guided tracking of fibers of the pyramidal tract combined with intraoperative cortical stimulation mapping in patients with gliomas. Journal of neurosurgery 101(1), 66-72 (2004)

[6] Sporns, O., Tononi, G., Kötter, R.: The human connectome: a structural description of the human brain. PLoS computational biology 1(4), 42 (2005)

[7] Griffa, A., Baumann, P.S., Thiran, J.-P., Hagmann, P.: Structural connectomics in brain diseases. Neuroimage 80, 515-526 (2013)

[8] Varoquaux, G., Craddock, R.C.: Learning and comparing functional connectomes across subjects. NeuroImage 80, 405-415 (2013)

[9] Guggisberg, A.G., Honma, S.M., Findlay, A.M., Dalal, S.S., Kirsch, H.E., Berger, M.S., Nagarajan, S.S.: Mapping functional connectivity in patients with brain lesions. Annals of neurology 63(2), 193-203 (2008) 
[10] Briganti, C., Sestieri, C., Mattei, P., Esposito, R., Galzio, R., Tartaro, A., Romani, G., Caulo, M.: Reorganization of functional connectivity of the language network in patients with brain gliomas. American Journal of Neuroradiology 33(10), 1983-1990 (2012)

[11] Kinno, R., Ohta, S., Muragaki, Y., Maruyama, T., Sakai, K.L.: Differential reorganization of three syntax-related networks induced by a left frontal glioma. Brain 137(4), $1193-1212(2014)$

[12] Harris, R.J., Bookheimer, S.Y., Cloughesy, T.F., Kim, H.J., Pope, W.B., Lai, A., Nghiemphu, P.L., Liau, L.M., Ellingson, B.M.: Altered functional connectivity of the default mode network in diffuse gliomas measured with pseudo-resting state fmri. Journal of neuro-oncology 116(2), 373-379 (2014)

[13] Garyfallidis, E., Brett, M., Amirbekian, B., Rokem, A., Van Der Walt, S., Descoteaux, M., Nimmo-Smith, I.: Dipy, a library for the analysis of diffusion mri data. Frontiers in neuroinformatics $\mathbf{8 ,} 8$ (2014)

[14] Tzourio-Mazoyer, N., Landeau, B., Papathanassiou, D., Crivello, F., Etard, O., Delcroix, N., Mazoyer, B., Joliot, M.: Automated anatomical labeling of activations in spm using a macroscopic anatomical parcellation of the mni mri single-subject brain. Neuroimage 15(1), 273-289 (2002)

[15] Deco, G., Tononi, G., Boly, M., Kringelbach, M.L.: Rethinking segregation and integration: contributions of whole-brain modelling. Nature Reviews Neuroscience 16(7), 430 (2015)

[16] Bullmore, E., Sporns, O.: Complex brain networks: graph theoretical analysis of structural and functional systems. Nature reviews neuroscience 10(3), 186 (2009)

[17] Rubinov, M., Sporns, O.: Complex network measures of brain connectivity: uses and interpretations. Neuroimage 52(3), 1059-1069 (2010)

[18] Rudie, J.D., Brown, J., Beck-Pancer, D., Hernandez, L., Dennis, E., Thompson, P., Bookheimer, S., Dapretto, M.: Altered functional and structural brain network organization in autism. NeuroImage: clinical 2, 79-94 (2013) 
[19] Drakesmith, M., Caeyenberghs, K., Dutt, A., Zammit, S., Evans, C.J., Reichenberg, A., Lewis, G., David, A.S., Jones, D.K.: Schizophrenia-like topological changes in the structural connectome of individuals with subclinical psychotic experiences. Human brain mapping 36(7), 2629-2643 (2015)

[20] Griffa, A., Baumann, P.S., Ferrari, C., Do, K.Q., Conus, P., Thiran, J.-P., Hagmann, P.: Characterizing the connectome in schizophrenia with diffusion spectrum imaging. Human brain mapping 36(1), 354-366 (2015)

[21] van den Heuvel, M.P., Fornito, A.: Brain networks in schizophrenia. Neuropsychology review $\mathbf{2 4}(1), 32-48(2014)$

[22] Gordon, E.M., Laumann, T.O., Adeyemo, B., Huckins, J.F., Kelley, W.M., Petersen, S.E.: Generation and evaluation of a cortical area parcellation from resting-state correlations. Cerebral cortex 26(1), 288-303 (2014)

[23] Hou, B.L., Bhatia, S., Carpenter, J.S.: Quantitative comparisons on hand motor functional areas determined by resting state and task bold fmri and anatomical mri for pre-surgical planning of patients with brain tumors. NeuroImage: Clinical 11, 378-387 (2016)

[24] Smith, S.M., Jenkinson, M., Johansen-Berg, H., Rueckert, D., Nichols, T.E., Mackay, C.E., Watkins, K.E., Ciccarelli, O., Cader, M.Z., Matthews, P.M., et al.: Tract-based spatial statistics: voxelwise analysis of multi-subject diffusion data. Neuroimage 31(4), $1487-1505(2006)$ 